Subscriber access provided by BUPMC - Bibliothèque Universitaire Pierre et Marie Curie

\title{
Article
}

\section{pH- and time-resolved in-situ SAXS study of self-assembled twisted ribbons formed by elaidic acid sophorolipids}

Prabhu Dhasaiyan, Sylvain Prévost, Niki Baccile, and Bhagavatula L V Prasad

Langmuir, Just Accepted Manuscript • DOI: 10.1021/acs.langmuir.7b03164 • Publication Date (Web): 19 Dec 2017

Downloaded from http://pubs.acs.org on December 22, 2017

\section{Just Accepted}

"Just Accepted" manuscripts have been peer-reviewed and accepted for publication. They are posted online prior to technical editing, formatting for publication and author proofing. The American Chemical Society provides "Just Accepted" as a free service to the research community to expedite the dissemination of scientific material as soon as possible after acceptance. "Just Accepted" manuscripts appear in full in PDF format accompanied by an HTML abstract. "Just Accepted" manuscripts have been fully peer reviewed, but should not be considered the official version of record. They are accessible to all readers and citable by the Digital Object Identifier (DOI®). "Just Accepted" is an optional service offered to authors. Therefore, the "Just Accepted" Web site may not include all articles that will be published in the journal. After a manuscript is technically edited and formatted, it will be removed from the "Just Accepted" Web site and published as an ASAP article. Note that technical editing may introduce minor changes to the manuscript text and/or graphics which could affect content, and all legal disclaimers and ethical guidelines that apply to the journal pertain. ACS cannot be held responsible for errors or consequences arising from the use of information contained in these "Just Accepted" manuscripts. 


\title{
pH- and time-resolved in-situ SAXS study of self-assembled twisted ribbons formed by elaidic acid sophorolipids
}

\author{
Prabhu Dhasaiyan, ${ }^{a}$ Sylvain Prevost, ${ }^{\mathrm{b}}$ Niki Baccile, ${ }^{* c}$ and Bhagavatula L. V. Prasad*a \\ ${ }^{a}$ Physical and Materials Chemistry Division, CSIR - National Chemical Laboratory, Pune - \\ 411008, India. Fax: (+91) 20-2590 2636; Tel: +91 202590 2013, \\ E - mail: pl.bhagavatula@ncl.res.in \\ ${ }^{\mathrm{b}}$ ESRF - The European Synchrotron, High Brilliance Beam line ID02, 38043 Grenoble, France \\ ${ }^{\mathrm{c}}$ Laboratoire de Chimie de la Matiere Condensee de Paris, Universite Pierre et Marie Curie, \\ 75252 Paris Cedex 05, France. Fax: 003314427 15 04, Tel: 0033144275677 , \\ E - mail: niki.baccile@upmc.fr
}

\begin{abstract}
:
Conditions that favor the helical structure formation in structurally similar sophorolipids (SLs), that is, elaidic acid SLs (having trans double bond between the C9 - C10 positions of the alkyl chain) and stearic acid SLs (no double bond) are presented here. The helical self - assembled structures formed by elaidic acid SLs were independent of $\mathrm{pH}$ and also were mediated by micellar intermediate. On the other hand, the stearic acid SLs formed helical structures under low $\mathrm{pH}$ condition only. Astonishingly, the formation routes were found to be different, albeit the molecular geometry of both the SLs is similar. Though a conclusive mechanistic understanding must await further work, our studies strongly point out that the non - covalent weak interactions in elaidic acid SL are able to overcome the electrostatic repulsions of the sophorolipid carboxylate groups at basic $\mathrm{pH}$ and facilitating the formation of helical structures. On the other hand, the hydrophobic interactions in stearic acid SLs endows the helical structures extra stability making them less vulnerable to dissolution upon heating.
\end{abstract}




\section{Introduction:}

Great strides have been made in our ability to map the structures of amphiphilic surfactant molecules to their self - assembling constructs and in leveraging such an understanding for a wide range of applications. ${ }^{1-3}$ Sophorolipids (SLs) are a class of bolaamphiphilic, glycolipid molecules derived by the fermentation action of yeast ( $S$. bombicola) on the fatty acids such as oleic acid, elaidic acid, linoleic acid etc. Due to the presence of a sophorose moiety at the hydrophobic end of the fatty acids, SLs have better solubility in water as compared to their pure fatty acid counterparts. Apart from that, SLs are proposed to have many potential applications such as antibacterial, anti-HIV, germicidal, antifungal, anticancer activities; low acute toxicity; sperm-immobilizing activities; and usefulness in dishwashing rinse aid formulations, template for the preparation of nanostructured silica, synthesis of water-dispersible metal nanoparticles etc. ${ }^{4,5,6}$ Furthermore, SLs also display a wide variety of self-assembled structures that are under deep investigation. Amongst the different SL molecules accessible, literature is predominantly devoted to the study of oleic acid based SLs [OASLs], ${ }^{7-11}$ which can form micelles or twisted ribbons according to the nature of the impurity and physico-chemical condition. ${ }^{12}$ Stearic acid sophorolipids (SASLs), which rather form ribbons, are by far the second most studied derivative. $^{13-15,16}$ On the other hand, studies on the elaidic acid based SLs (EASLs) are very scant. ${ }^{16}$ Thus, it may not be very surprising to note that our understanding of the molecular structure - self-assembly dependence is still in its infancy, as far as these bolaamphiphilic systems are concerned, because the systematic research into their self-assembling structures started appearing only in the last decade or so. Very recently, a few proposals to understand such a dependence by considering the horseshoe-like architecture of SLs, or the melting points of their lipidic part have been put forward. ${ }^{17,18,19}$ However, there is still a long way to go as far as complete understanding of the SL self-assemblies are concerned, as we show in this study by looking at the behaviour of two structurally similar SL based bolaamphiphiles, namely, elaidic acid SL (EASL) and stearic acid SL (SASL).

EASL and SASL are characterized by a linear C18 fatty acid, where the main difference is the presence of trans-9 double bond in EASL, and in this sense they can be considered the exact glycosydic counterpart of stearic and elaidic acids. Considering the structural proximity between EASL and SASL, one does not expect major differences in their self-assembly process on the basis of the packing parameter alone, which is reasonably expected to be equal for both 
molecules, the packing parameter being the well-known and generally-recognized standard that associate the molecular geometry to the equilibrium self-assembled morphology. ${ }^{20}$ In fact, the strong molecular similarity of these two SLs can certainly explain the similarity in their selfassembly process, as both form twisted ribbons. ${ }^{13,16}$ However, if EASL assembles into ribbons in the entire $\mathrm{pH}$ range, SASL only forms ribbons below $\mathrm{pH} \sim 7.2$ and micelles at basic $\mathrm{pH} .{ }^{13,18}$ The kinetics of self-assembly also seems to be very different. Ribbon formation for EASL varies from slow to very slow at, respectively, acidic and basic $\mathrm{pH}$, while it is immediate at acidic $\mathrm{pH}$ for SASL.

Helical structures ${ }^{21-24}$ are not only important in biological systems (DNA), but also in applications such as enantioselective catalysis, chiral recognition, chiral catalysis, helical crystallization of macromolecules ${ }^{25-27}$ and their formation is important in the process of understanding Alzheimer disease. ${ }^{28-30}$ In addition to this aspect, trans fats can have a great impact on cell walls/ biological membranes and are implicated in the appearance of dietary health diseases going from coronary heart disease to insulin sensitivity, and even cancer. ${ }^{31-33}$ Unfortunately, the only studies focused on the self-assembly properties of the fatty acids (elaidic and stearic) corresponding to EASL and OASL, and onto which one could rely on to better understand the data concerning EASL and OASL, were not carried out on the molecules themselves, but by mixing them with phosphatidylcholine lipids or monoolein/elaidins. If such works have generated tremendous curiosity, several ambiguities still exist, with a gross consensus that trans fatty acids behave similarly to saturated fatty acids. In particular, their inclusion in model bilayers, ${ }^{34,35}$ or the use of phospholipids containing oleic, stearic or elaidic tails $^{36}$ show that cis geometry favours more fluid membranes, or mesophases bearing a higher curvature. Although these studies are very important to better understand the impact of trans fats in biological membranes, they do not offer much help with respect to our understanding regarding the interdependence of molecular stereochemistry and their self-assemblies. In the context of pure fatty acids, only few studies exist in this sense and they mainly concern the selfassembly of oleic acid (18:1 cis-9) only. ${ }^{37}$ For instance, the group of S. R. Raghavan illustrated that sodium oleate $(\mathrm{NaOA})$ forms $\mathrm{pH}$ sensitive vesicles $($ at $\mathrm{pH}$ higher than $\sim 10$ ) that undergo a transition from vesicles to micelles as the $\mathrm{pH}$ is increased. ${ }^{38}$ When it comes to the comparison of oleic to elaidic and stearic acids, little, if nothing, can be found. In the context of membrane fluidity mentioned above, Yaghmur et al. ${ }^{35}$ stated that the different impact of oleic compared to 
elaidic acid depends on the difference in the relative value of their packing parameter. Despite the same number of carbon atoms in the tail, oleic acid is supposed to have a shorter effective chain length with respect to elaidic acid due to the "kink" introduced by the cis double bond at the position 9, and for this reason curved bilayers are favoured. In practice, a closer look at the work of Yaghmur also shows the importance of hydration of the carboxylic group, the temperature, as well as the mutual interactions between the fatty acids and the monoolein and monoelaidin-constituted mesophase. For this reason, we believe that any conclusion about a clear relationship between the molecular shape and the final mesophase cannot be drawn from their work.

With this background, we set out to explore the impact of the trans mono unsaturation in the fiber-forming properties of EASL by comparing it to the self-assembly properties of its structural analogue, SASL, by mean of in-situ Small Angle X-ray Scattering (SAXS) and Light Scattering (LS) under various conditions ( $\mathrm{pH}$, temperature). We believe that this not only provides more insights into the complex field of self-assembled helical structures, but also clarifies the selfassembly properties of similar sophorolipids, thus giving a step forward in their utilization for their widespread applications. ${ }^{4,6,39-41}$

\section{Materials and Methods:}

The chemicals used for MGYP media were purchased from Sigma - Aldrich. This comprises of Maltose, Glucose, Yeast (non - pathogenic) and Peptone. The water used for the whole experiments are purified by Milli Q system with a resistivity of $18.2 \mathrm{M} \Omega \mathrm{cm}$. Elaidic acid, $\mathrm{NaOH}$ pellets were also purchased from Sigma - Aldrich and used as received without any further purification. All solvents used for the entire experiments are analytical grade (AR). HCl was procured from Merck. Each time, a fresh solution of $0.1 \mathrm{M} \mathrm{HCl}$ and $5 \mathrm{M}$ were used throughout the experiments.

Preparation of self - assembled structures. The self - assembled structures was prepared by $\mathrm{pH}$ variation method. It involves the following steps. Step 1:1 mL of Milli Q water was taken in a clean glass sample vial; step 2: weighed amount of SL (5 mg) powder was added to $1 \mathrm{~mL}$ of water $(0.5 \mathrm{wt} \%)$; step 3: the $\mathrm{pH}$ of the solution was increased to basic $(\mathrm{pH} \sim 11)$ by adding $10-20$ $\mu \mathrm{L}$ of a $5 \mathrm{M} \mathrm{NaOH}$ solution and adjusted (decreased) to the desired value by adding $\mu \mathrm{L}$ amounts of freshly prepared $0.1 \mathrm{M} \mathrm{HCl}$. 
Scanning Electron Microscopy (SEM). The details of self - assembled structures of elaidic acid SLs were unraveled using SEM. A drop $(60-70 \mu \mathrm{L})$ of solution containing self - assembled structure were drop casted on silicon wafer which were pre - cleaned thoroughly with Piranha solution (1:3 ratio of $30 \% \mathrm{H}_{2} \mathrm{O}_{2}$ and con. $\left.\mathrm{H}_{2} \mathrm{SO}_{4}\right)$ and the samples were air dried overnight. Since SLs are non-conducting surfactant type molecules, prior to SEM imaging the samples were sputter coated with $7 \mathrm{~nm}$ gold ( $\mathrm{Au}$ ) film using a Polaron SC 6420 sputter coater. The self assembled structures were imaged using Quanta 200 3D scanning electron microscope (SEM) equipped with a tungsten filament gun, operating at WD $10.6 \mathrm{~mm}$ and $20 \mathrm{kV}$.

Cryogenic transmission electron microscopy (Cryo -TEM). Transmission electron microscopy experiments under cryogenic conditions (cryo - TEM) were carried out on an FEI Tecnai 120 Twin microscope operating at $120 \mathrm{kV}$ equipped with a high resolution Gatan Orius CCD numeric camera. The sample holder was a Gatan Cryo holder (Gatan 626DH, Gatan). Digital Micrograph $^{\mathrm{TM}}$ software was used for image acquisition. Cryo fixation was done on a homemade cryo-fixation device. The solutions were deposited on glow discharged holey carbon coated TEM copper grids (10 $\mu \mathrm{m}$, Quantifoil R2/2, Germany). For analysis $5 \mu \mathrm{L}$ of the self - assembled structure were drop casted on the TEM grid. The excess solution was removed and the grid was immediately plunged into liquid ethane at $-180{ }^{\circ} \mathrm{C}$ before transferring them into liquid nitrogen. All grids were kept at liquid nitrogen during storage and throughout all experimentation. This was performed using the parent solution few minutes before cryo-fixation. All grids were stored under liquid nitrogen until used for image acquisition.

Small Angle X-ray Scattering (SAXS). Time-resolved in-situ Small Angle X-ray Scattering (SAXS) experiments have been done using a flow-through polycarbonate $2 \mathrm{~mm}$ capillary connected to the sample-containing solution at $\mathrm{pH} \sim 11$ through a peristaltic pump. The $\mathrm{pH}$ was controlled in situ via a classical $\mathrm{KCl}$ pH-meter directly located in the experimental hutch and monitored in real time. $\mathrm{pH}$ changes have been obtained by using a $0.1 \mathrm{M} \mathrm{HCl}$ solution introduced via a motor-controlled syringe pump. ${ }^{18}$ Data have been acquired on the high brilliance ID02 beam line at the ESRF synchrotron (Grenoble, France). The sample-to-detector distance was set to $1 \mathrm{~m}$ and the X-ray beam energy at $12.6 \mathrm{keV}$. Each spectrum was collected for $1 \mathrm{~s}$. A CCD camera was used to collect the scattered photons and integrated azimuthally to obtain a typical I(q) spectrum. Contribution of the solvent (water) and capillary have been measured prior to the 
experiment and duly substracted during the data treatment. All data have been corrected for the transmission of the direct beam and scaled to be in absolute scale.

SAXS data modeling using a core shell ellipsoid of revolution form factor has been extensively described in recent works and it will not be detailed here. ${ }^{10,18}$ The entire series of time-resolved experiments has been modelled between $0.42<\mathrm{q}\left[\mathrm{nm}^{-1}\right]<1.84$ so to leave out the upcoming diffraction peak, but modelling on a larger q-scale $\left(0.42<\mathrm{q}\left[\mathrm{nm}^{-1}\right]<4.50\right)$ was also successfully tested (see supporting information, modelling of the time-resolved in-situ SAXS data section). To control the fit at best, we have set the core Scattering Length Density (SLD), $\rho_{\mathrm{c}}$, to $8.3 \times 10^{-4}$ $\mathrm{nm}^{-2}$ and the solvent $\rho_{\text {solv }}$ to $9.4 \times 10^{-4} \mathrm{~nm}^{-2}$ as fixed parameters, disregarding the possibility of solvent penetration at the sophorose/fatty acid palisade. ${ }^{10}$ The volume fraction is set at $0.5 \mathrm{w} \%$. The overall quality of the fit is followed by the classical $\chi^{2} / \mathrm{Npts}$ evolution test.

Dynamic Light Scattering measurements (DLS). The light scattering measurements were done on a Malvern Zetasizer Nano - ZS 90 instrument $(\lambda=633 \mathrm{~nm})$ at constant shutter opening and same sample-to-detector distance and a $90^{\circ}$ geometry. Zetasizer software was used to analyze the results. The scattered light is expressed in terms of the derived count rate (DCR) in kilo counts/seconds (kcps). In order to minimize effects of dust and maximize the reproducibility, each sample was analyzed 3 times. Each analysis is characterized by 20 measurements of $5 \mathrm{~s}$ each. For the heating experiments, the samples were heated internally without taking out the cuvette.

Raman Spectra. Raman spectra were recorded using a Hololab 5000 R apparatus (Kaiser Optical Systems, Inc.) equipped with a $785 \mathrm{~nm}$ laser (power $=10-12 \mathrm{~mW}$, accumulation time $=15 \mathrm{~s}$ and resolution $=4 \mathrm{~cm}^{-1}$ ) connected to a microscope equipped with a $\mathrm{x} 50$ lens.

Preparation of Sophorolipids. EASL were prepared based on our previous reports using glucose and elaidic acid in presence of Candida bombicola. ${ }^{16}$ The fermentation medium consists of glucose (100 g), yeast extract (10 g), peptone $(0.6 \mathrm{~g})$ and elaidic acid ( 10 g) in $1 \mathrm{~L}$ of milliQgrade water. After 7 days of fermentation, the crude sophorolipid mixture was extracted three times using ethyl acetate and the excess solvent was removed under reduced pressure in a rotavapor. The crude SLs were separated from the unreacted fatty acids by extracting the latter with hexane. The stearic acid SLs was derived by the reduction reaction of crude oleic acid SLs 
(from the previous batches) with $\mathrm{Pd} / \mathrm{C}$ in methanol using hydrogen balloon pressure. The reaction was allowed to proceed for $6 \mathrm{~h}$ without any disturbance. The completion of the reaction was monitored by TLC. After the completion of reaction, the Pd metal was filtered through G3 sintered funnel and washed with $\mathrm{MeOH}$. The filtrate methanolic solution was collected in a separate $500 \mathrm{~mL}$ round bottom flask. The crude stearic acid SLs was obtained by removing the solvent under reduced pressure in a rotavapor. Simple alkaline hydrolysis of the crude mixture of SLs with $5 \mathrm{M} \mathrm{NaOH}$ solution followed by $3 \mathrm{~h}$ refluxion provides access to acidic form of respective SLs. The hydrolyzed acidic sophorolipid was further purified by silica gel column chromatography using chloroform/methanol mixture (80:20) as the eluting system and it was further submitted to Soxhlet treatment using hot hexane. The visualization of TLC was done with phosphomolybdic acid (PMA) by warming the substrate. Finally, the acidic SLs were characterized by ${ }^{1} \mathrm{H}$ NMR using $\mathrm{CD}_{3} \mathrm{OD}: \mathrm{CDCl}_{3}$ (1:1). ${ }^{1} \mathrm{H}$ spectra were recorded on a Bruker

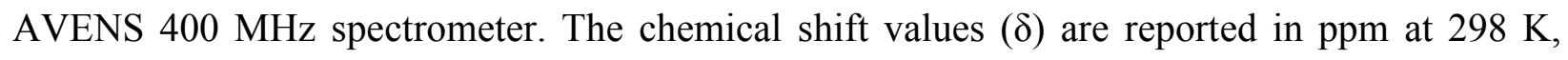
with trace amount of tetramethylsilane (TMS, $0 \mathrm{ppm}$ ) as internal standard. According to the NMR analysis, the final EASL compound contains about $5 \mathrm{~mol} \%$ of residual fatty acids (characteristic peak at $\delta=0.92 \mathrm{ppm}$, Figure S1), with a EASL/OASL (OASL= Oleic Acid Sophorolipids) ratio of about $80 / 20$, where the signals at $\delta=5.40 \mathrm{ppm}$ and $\delta=5.36 \mathrm{ppm}$ can be respectively attributed to EASL and OASL (Figure S2b).

\section{Results:}

The typical chemical structure of elaidic and stearic acid SLs are shown in Figure 1, the synthesis of which was reported in earlier work. ${ }^{13,16}$ The ${ }^{1} \mathrm{H}$ NMR signature of SASL, which is obtained by catalytic hydrogenation of the oleic acid SL, is very similar to the former, except for the lack of the $\mathrm{C} \underline{H}=\mathrm{CH}$ double bond signal at $5.37 \mathrm{ppm}$. The homogeneity of this sample has been assessed before. ${ }^{13,16}$ On the contrary, EASL is obtained by the direct metabolism of $S$. bombicola on elaidic acid and glucose as carbon sources. To dissipate any doubt that this compound is mainly constituted of EASL, we performed both a ${ }^{1} \mathrm{H}$ NMR and Raman spectroscopy analysis of the EASL sample in solution and solid state. Both these techniques provided an unambiguous proof for the presence of a majority (about $80 \mathrm{~mol} \%$ ) of EASL. The ${ }^{1} \mathrm{H}$ NMR spectrum of EASL (Figure S1) shows, besides the typical signatures of the fatty acid $(2.5<\delta /$ ppm $<1.0)$ and sophorose $(4.6<\delta /$ ppm $<3.0)$ backbone, ${ }^{16,42}$ a downfield shift of the 
$\mathrm{C} \underline{H}=\mathrm{CH}$ double bond by $0.3 \mathrm{ppm}$, as expected for trans isomers. ${ }^{43}$ Although this proof could be enough, we have also collected the corresponding Raman signature, which is known to be extremely sensitive to the $\mathrm{C}=\mathrm{C}$ stretch and selective between the cis $\left(1655 \mathrm{~cm}^{-1}\right)$ and trans $(1670$ $\mathrm{cm}^{-1}$ ) isomers. ${ }^{44}$ More details are given in the Supporting Information section.

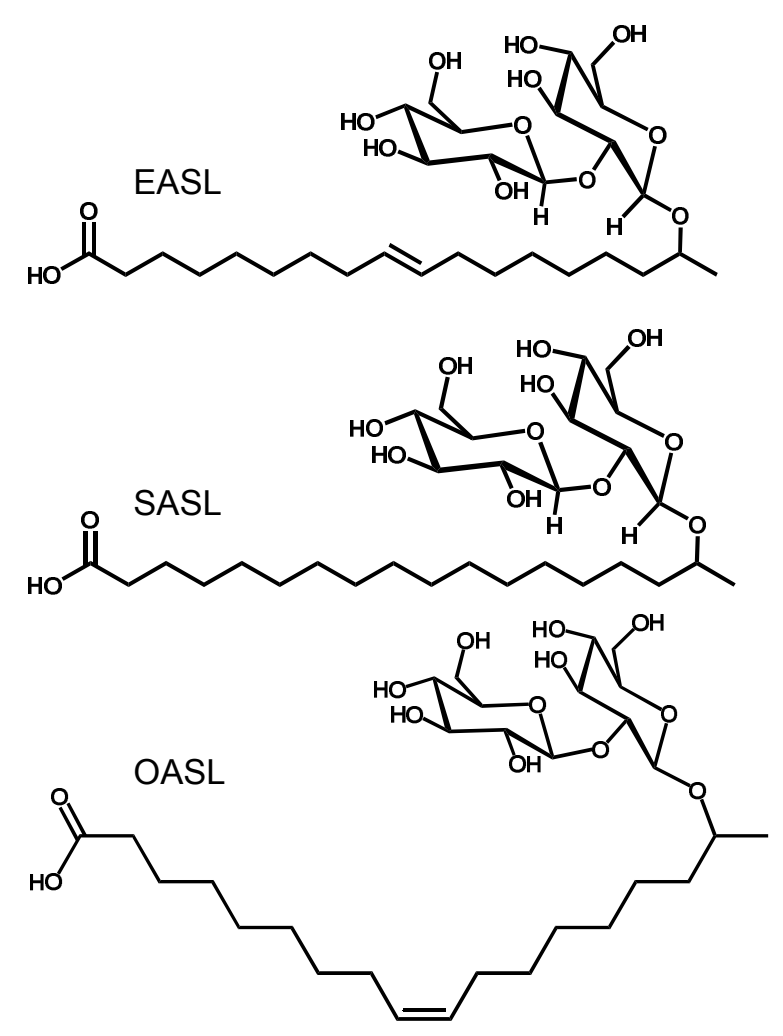

Figure 1: The chemical structures of monounsaturated trans C18:1(EASL) and saturated C18:0 (SASL) molecules used in the present study. For comparison, the structure of the most studied cis C18:1(OASL) is also displayed. 

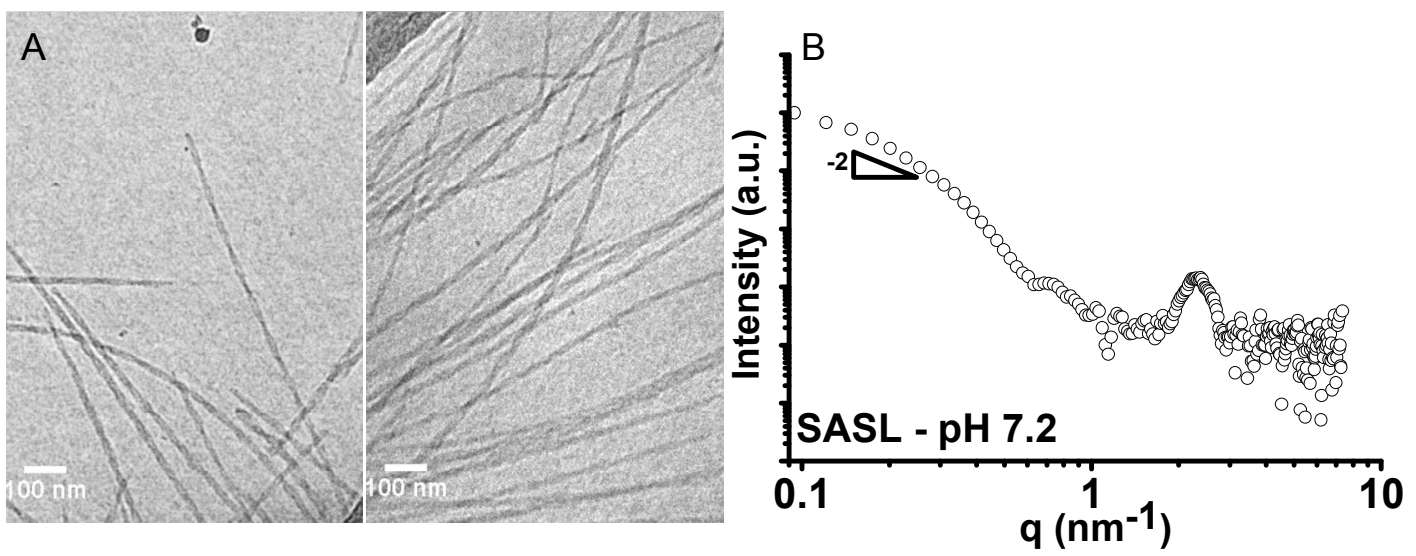

Figure 2: (A) Cryo - TEM image and (B) SAXS profile of SASLs at conditions where they form helical structures (experiments are performed at room temperature, $25^{\circ} \mathrm{C}$ ).

The $\mathrm{pH}$ dependent self - assembled structures of SASL have been reported in detail by some of us $^{13-16}$ and therefore only the most pertinent results are mentioned here. Briefly, at basic $\mathrm{pH} \sim 11$, SASLs form a clear solution. SAXS analysis of this clear solution reveals the presence of micellar phase. As the $\mathrm{pH}$ is decreased to $\mathrm{pH}=7.4 \pm 0.1$, the solution becomes instantaneously turbid (Figure S3A). An aliquot of this sample reveals the presence of twisted fibers (Figure 2A) in agreement with the previous report. The SAXS analysis of this sample, presented in Figure 2B on an aliquot prepared at $\mathrm{pH} 7.2$, shows the classical signature of a ribbon: ${ }^{45-47}$ at $\mathrm{q}<0.4 \mathrm{~nm}^{-1}$, the $\log -\log$ plot of the intensity follows the trend $\mathrm{I}(\mathrm{q}) \sim \mathrm{q}^{-2}$, in agreement with the previous report ${ }^{13}$ and with complementary visual characterization (cryo-TEM in Figure 2A). At $q=2.32$ $\mathrm{nm}^{-1}$, a broad Bragg peak characterizes the SAXS profile. The corresponding interplanar distance $\mathrm{d}=2.71 \mathrm{~nm}$ was previously attributed to the lateral inter-lipid layer distance within each ribbon. ${ }^{13}$ If the broad nature of the peak strongly suggests a crystalline packing (to which we will refer for the rest of this work) of SASL on distances corresponding to few nanometers, as expected by the Scherrer equation, one cannot exclude an ordered liquid crystalline, instead, as postulated by other works ${ }^{48-50}$ and discussed in detail in ref. 51 . 

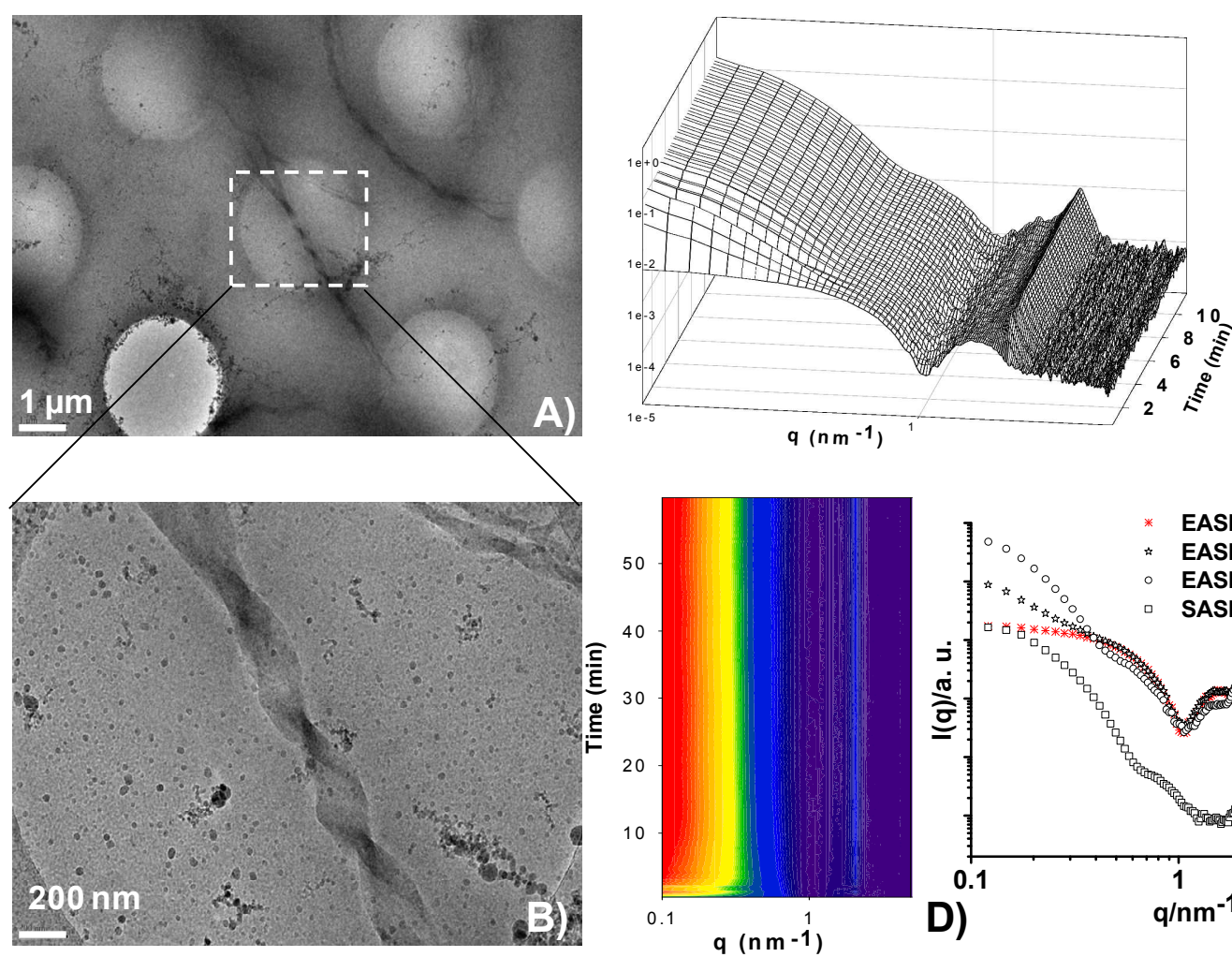

C)
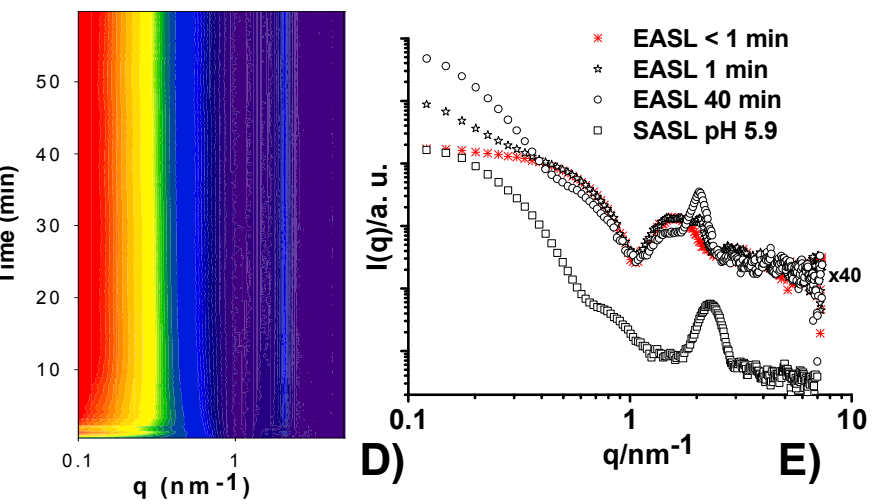

Figure 3: (A, B) cryo - TEM images of self - assembled structure of EASLs at $\mathrm{pH}=6.7$ and room temperature $\left(25^{\circ} \mathrm{C}\right)$. (C) Side view and (D) contour plot of the time-resolved in-situ SAXS pattern of EASLs at $\mathrm{pH}=6.7$ and room temperature $\left(25^{\circ} \mathrm{C}\right)$. (E) Comparison between SAXS patterns recorded for SASL (empty squares, $5 \mathrm{mg} / \mathrm{mL}, \mathrm{pH} 5.9)$ and EASL $(5 \mathrm{mg} / \mathrm{mL}, \mathrm{pH}=6.7)$ at $\mathrm{t}<1$ min (red stars), $\mathrm{t}=1 \min$ (black stars) and $\mathrm{t}=40 \mathrm{~min}$ (open circles).

In the case of EASLs, the trend of $\mathrm{pH}$ dependent scattering is significantly different compared to the behaviour of SASLs. Initially dissolving the EASL in water at all $\mathrm{pH}$ ranges leads to the formation of clear solution, which becomes slightly turbid upon standing for some time. At $\mathrm{pH}$ below 6, mild turbidity appears in the range of about $20 \mathrm{~min}$, and it strongly increases after about $30 \mathrm{~min}$ to $1 \mathrm{~h}$. At $\mathrm{pH}$ above 9, the turbidity appears only after $30 \mathrm{~min}$ and one needs to wait $\sim 1 \mathrm{~h}$ for a highly turbid solution. This behaviour is very different than SASL, in which the micellar phase (observed at $\mathrm{pH}>7.4)^{18}$ does not undergo any phase change. As can be seen, these qualitative observations in fact suggest that $\mathrm{pH}$ has a less drastic influence on the self-assembly properties of EASL in solution. ${ }^{16}$ 
The quantitative analysis of the process in case of EASL are presented hereafter. First of all, one must analyze the nature of the turbid form that results both under acidic and basic conditions. Cryo - TEM and SEM reveal vivid helical structures both at neutral $(\mathrm{pH}=6.7)$ (Figure $3 \mathrm{~A}, \mathrm{~B})$ and basic $\mathrm{pH}$ (Figure $4 \mathrm{~A}, \mathrm{~B}$ ). The helical fibers have length in the range of few hundred $\mu \mathrm{m}$, width is in between $2-4 \mu \mathrm{m}$, thickness of $400-800 \mathrm{~nm}$, and the pitch lies between $10-12 \mu \mathrm{m}$ under acidic conditions and 2-10 $\mu \mathrm{m}$ under basic conditions. Between the EASL and SASL (Figure 2A and 4A, B, respectively), while the length of helical structures is comparable to each other, ranging from several hundred nanometer to several microns, the side width of SASL structures is rather small (in the order of $15 \mathrm{~nm}-30 \mathrm{~nm}$ on the most polydisperse samples and $13.5 \pm 1.5 \mathrm{~nm}$ on the most uniform ones). ${ }^{13,14}$ The pitch was also found to be between $150 \mathrm{~nm}$ and $200 \mathrm{~nm}$. In comparison, EASL ribbons are at least two orders of magnitude wider and one order of magnitude longer pitch, (at all $\mathrm{pH}$ values). Thus, all these analyses makes it very clear that the structural characteristics of the EASL ribbons strongly differ from the ones measured on the SASL ribbons.

We have recently employed $\mathrm{pH}$-resolved in-situ SAXS to follow the local structural variations in the micelle-to-ribbon transition in $\mathrm{SASL}^{18}$ and we have found that micelles seem to behave as a reservoir of matter, and not as nucleation centers for the ribbon formation. We have applied this strategy to the time-dependent fiber-formation of EASL both under acidic and basic $\mathrm{pH}$ conditions. Time-resolved in-situ SAXS experiments are shown in Figure 3C, D and Figure 4C for, respectively, acidic and basic media. The SAXS data recorded on EASL under acidic conditions (Figure 3C, D) show a typical micelles signal only in the very initial stages of the experiment, below $\sim 1 \mathrm{~min}$, followed by a fast-appearance of a typical scattering signal of the ribbons. ${ }^{a}$ The perspective view of the SAXS data (Figure 3C) shows that the ribbon signal is practically concomitant with the micelles with little or no transition between them; a Bragg diffraction peak is observed at $\mathrm{q}=2.06 \mathrm{~nm}^{-1}$ and the top-view of the SAXS experiments (Figure 3D) clearly shows its stability over time, that is, no time-dependent variation in the interplanar distance occurs, a fact which indicates that once the ribbons are formed, their crystalline

\footnotetext{
${ }^{a}$ Please note that this time scale is at least 20 times shorter than what is observed and described for SASL system. In fact, we must outline that SAXS data are recorded under dynamic conditions, where the sample is constantly pumped through the capillary by a peristaltic pump. We repeated the experiment several times and we systematically observed faster formation of the ribbons under the dynamic conditions as compared to the static bench conditions.
} 
structure is stable. The calculated d-spacing is $3.05 \mathrm{~nm}$, about $12 \%$ higher than the value found on the SASL ribbon structure. One can estimate the size of sophorolipid with a fully extended fatty acid chain to be above $3 \mathrm{~nm}$, if one counts about $2.2 \mathrm{~nm}$ for the fatty acid using the Tanford formula and the size of sophorose being in the order of $1 \mathrm{~nm} .{ }^{10}$ By extending the hypothesis according to which the packing within the EASL ribbons is analogous to the one found in the SASL ribbons, one could suppose that the bending angle of the monolayer lipid membrane is smaller than what was found in that system (about $33^{\circ}$ ), ${ }^{13}$ that allows it to achieve the higher dspacing. By closely looking at the EASL SAXS patterns recorded in the first minute of the reaction (Figure 3E), one can identify both the classical scattering pattern of a spherical/ellipsoidal sophorolipid micelles, ${ }^{10,18}$ as one can suppose from the form factor oscillation in the $1<\mathrm{q}\left[\mathrm{nm}^{-1}\right]<3$. In the same order of time scale, one can observe an additional contribution coming from the ribbons, as indicated by the low-q scattering intensity and small diffraction peak at $\mathrm{q}=2.06 \mathrm{~nm}^{-1}$ (red stars in Figure 3E). Interestingly, after 40 minutes, the SAXS signal still contains contribution from the micelles scattering, but an important scattering in the low-q region and a more pronounced diffraction peak now characterize the presence of twisted ribbons in larger amounts.

The time-resolved in-situ SAXS experiment on EASLs performed under basic conditions is presented in Figure 4C, D. A quick look at the time evolution of the SAXS profiles in Figure 4C confirms the slower kinetics of the ribbon formation, as compared to the acidic medium (Figure 3C); this is coherent with the ex-situ observation under static conditions. The system is characterized by the typical scattering signal of the micelles from time, $t=0$ until $t \sim 40$ min, after which the low-q (below $0.5 \mathrm{~nm}^{-1}$ ) scattering signal starts to increase and the inter-lipid diffraction peak appears. The intensity of the latter continuously grows between $\sim 40<\mathrm{t} / \mathrm{min}<$ $\sim 60$, and it becomes stable towards the end of the experiment, stopped after $100 \mathrm{~min}$. Interestingly, the peak position is exactly the same as the one found in the acidic medium, $\mathrm{q}=$ $2.06 \mathrm{~nm}^{-1}$, thus indicating that the crystal structure of the ribbons is the same and confirming that $\mathrm{pH}$ has no major influence on the nature of the self-assembled structure. Similarly as in the acidic medium, the SAXS signal of the ribbons always contains a non-negligible fraction of the micelles. Micelles are commonly found in sophorolipids systems and we have previously modeled the SAXS data of both OASL and SASL ${ }^{18}$ as a function of $\mathrm{pH}$. On the basis of that work, we employ here the same methodology and we apply a core-shell ellipsoid form factor 
model to fit the time-resolved in-situ SAXS data. This model is only meant to follow the micellar portion of the SAXS data and it considers that the core of the micelle is composed of the elaidic acid moiety and the shell is composed of hydrated sophorolipids. We operate a best-fit procedure on the first scattering profile at $\mathrm{t}=0$ and then we let free the most sensitive parameters of the fit for the entire time-resolved series. Considering the fact that the system is composed of two distinct phases, we only model the micellar region between $0.44 \mathrm{~nm}^{-1}$ and $1.79 \mathrm{~nm}^{-1}$, which includes the micellar oscillation but it excludes the strong low-q scattering and the diffraction peak at $\mathrm{q}=2.06 \mathrm{~nm}^{-1}$. Two model-independent fits are also done in parallel: we measure the slope of the low-q portion $\left(0.15-0.66 \mathrm{~nm}^{-1}\right)$ of the profile, which accounts for the morphological evolution on large scales, and the radius of gyration, Rg. Figure 4D reports the time-evolution of $\mathrm{Rg}$ and the low-q, while Figure S4 reports the time evolution of three model-dependent parameters: the shell thickness, $\mathrm{T}_{\mathrm{s}}$, the core radius, $\mathrm{R}_{\mathrm{c}}$ and the shell Scattering Length Density, $\rho_{\mathrm{s}}$, including the value of $\chi^{2}$.

First of all, at time $\mathrm{t}=0, \mathrm{Rg}$ is about $3.3 \mathrm{~nm}$ while at $\mathrm{t}=100 \mathrm{~min}, \mathrm{Rg}=3.5 \mathrm{~nm}$, thus indicating that $\mathrm{Rg}$ is practically constant with time. Meanwhile, the time evolution of the low-q slope shows a gradual transition from a pure micellar system (no slope) to fibers (I (q) $\sim q^{-2}$ ) in the same timespan; one can also affirm that it takes about $40 \mathrm{~min}$ to reach the vicinity of -2 , a time-scale which is also needed to display the appearance of the inter-lipids diffraction peak. Nonetheless, one must also observe that the micellar signal still lay underneath the ribbon signal. Therefore to understand this aspect better, we compare the micellar phase observed in case of the most studied oleic acid sophorolipids (OASL, Figure 1) with that observed with the present system, namely EASL. Interestingly, if one compares the SAXS profile of a typical OASL sample at acidic $\mathrm{pH}$ (4.2) with the SAXS profile of EASL recorded both at acidic and basic $\mathrm{pH}$ at $\mathrm{t}=0 \mathrm{~s}$ (Figure $\mathrm{S} 4 \mathrm{a}$ ), one can clearly observe that both EASL spectra are very similar but notably shifted to the high-q region, and having a remarkably different oscillation in the first minimum of the form factor. These facts suggest that the micellar structure of EASL is very similar at both acidic and basic $\mathrm{pH}$ and, above all, that is very different than what was described for OASL. ${ }^{10,18}$ If we apply the same model function used for OASL to fit EASL data, we obtain two sets of possible solutions. In the first one, reported as Model 1 in Table 1, we impose a unitary aspect ratio both for the shell and core regions (core-shell sphere), while in Model 2, we let these parameters free (coreshell ellipsoid of revolution). 

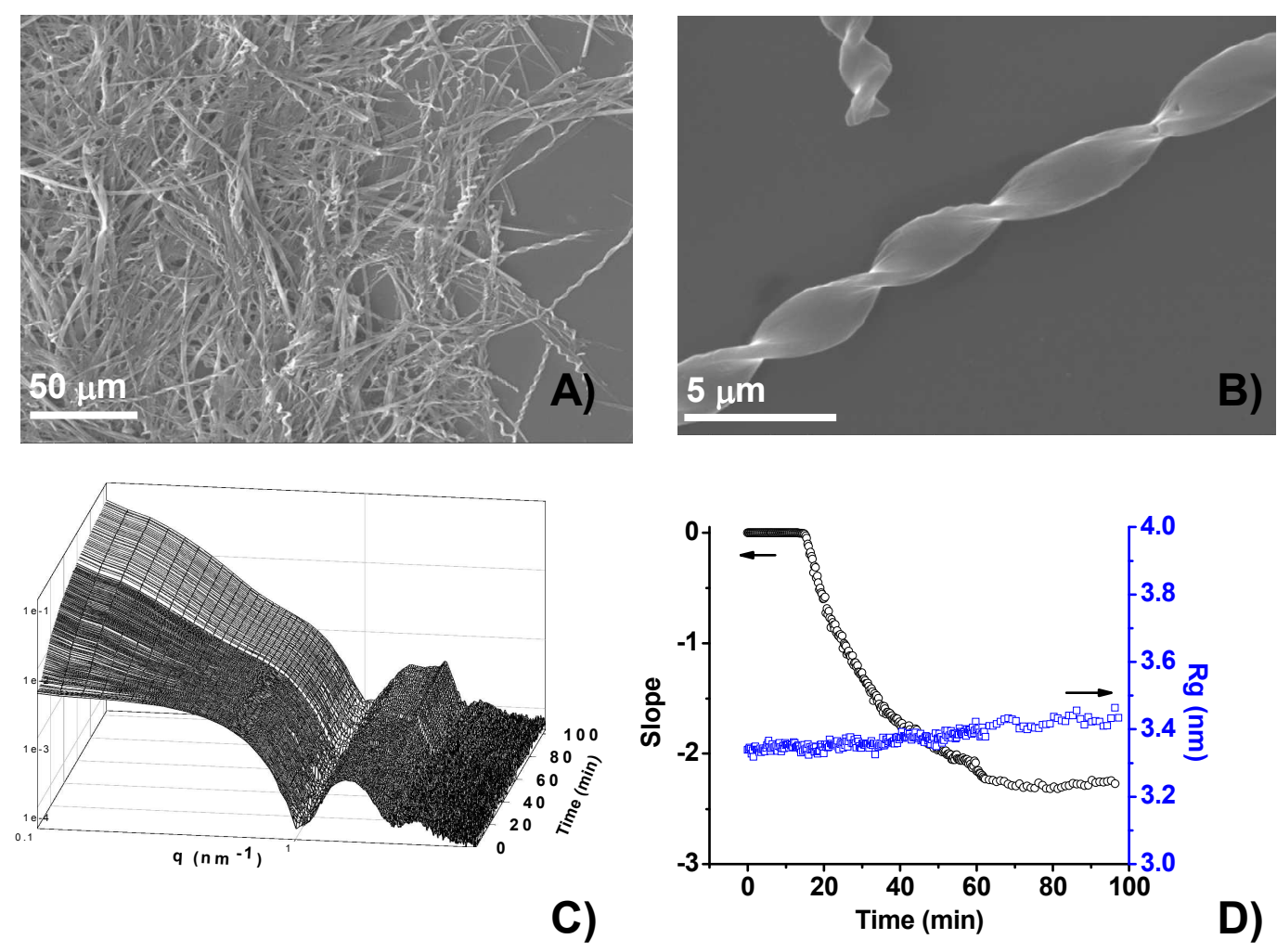

Figure 4: (A, B) SEM images of self - assembled structure of EASLs at $\mathrm{pH} 11$ and room temperature $\left(25^{\circ} \mathrm{C}\right)$. (C) Time-resolved in-situ SAXS pattern of EASLs at $\mathrm{pH} 11$ and room temperature $\left(25^{\circ} \mathrm{C}\right)$. (D) Time-dependency of the model-independent slope $\left(\log (\mathrm{I})\right.$ vs. $\log (\mathrm{q})$, measured at $\left.\mathrm{q}<0.35 \mathrm{~nm}^{-1}\right)$ and radius of gyration, $\mathrm{Rg}$, extracted from the time-resolved $\mathrm{SAXS}$ data from $\mathrm{t}=0$ and $\mathrm{t}=100 \mathrm{~min}$ at basic $\mathrm{pH}$.

According to the results obtained from model 1 , one can find a value for $R_{c}$ of $1.15 \mathrm{~nm}$, a value for $T_{S}$ of about $2.34 \mathrm{~nm}$ and $\rho_{\mathrm{S}}$ of about $9.67 \times 10^{-4} \mathrm{~nm}^{-2}$. First of all, these values are practically constant over time and $\mathrm{pH}$, in agreement with $\mathrm{Rg}$. This means that the micelles can be modeled as extremely stable (under any $\mathrm{pH}$ conditions) core-shell spheres and they do not evolve with time. The typical fitting function can be found on Figure S4a. If this model seems to be appropriate, one should look at the absolute values for $\mathrm{R}_{c}, \mathrm{~T}_{\mathrm{S}}$ and $\rho_{\mathrm{s}}$. If compared to the OASL system, ${ }^{18} \mathrm{R}_{\mathrm{c}}$ can be considered comparable within a $30 \%$ range, as this is generally found below $1 \mathrm{~nm}$. However, $\mathrm{T}_{\mathrm{s}}$ was found to vary between 0.5 (basic $\mathrm{pH}$ ) and 1.2 (acidic $\mathrm{pH}$ ) while $\rho_{\mathrm{s}}$ is generally found to be above $10 \times 10^{-4} \mathrm{~nm}^{-2}$. According to Model 1, $T_{\mathrm{S}}$ is about five times larger at basic $\mathrm{pH}$ and two times larger at acidic $\mathrm{pH}$ compared to the same values of OASL. According to 
the hypothesis that the shell thickness is composed of hydrated sophorose, the size of which is in the order of $1 \mathrm{~nm}$, we decided to take these results with caution and try a second model.

In Model 2, we have set a higher value of $R_{c}$ and $\rho_{s}$ and a lower value of $T_{S}$ as starting parameters. In order to obtain a different set of results, we also set the core and shell aspect ratios as free parameters, as the results obtained in Model 1 are systematically obtained, otherwise. All in all, Model 2 considers that the sophorose shell is in the order of the expected size of sophorose and the core radius corresponds to the actual size an elaidic acid molecule, instead of a set of interpenetrated molecules, as found in $\mathrm{OASL}^{10,13}$ and as predicted by Nagarajan ${ }^{52}$ for bolaform surfactants. The results of the fit for Model 2 (Table 1) indicate an $R_{c}$ between $2.42 \mathrm{~nm}$ and 3.03 $\mathrm{nm}$, a small $\mathrm{T}_{\mathrm{S}}$ comprised between $0.68 \mathrm{~nm}$ and $1.06 \mathrm{~nm}$ and a practically constant $\rho_{\mathrm{S}}$ at $9.67 \times 10^{-}$ ${ }^{4} \mathrm{~nm}^{-2}$. If these values could suggest a micellar structure, that is more classically found in nonbola surfactant systems, one should note the values for the core and shell aspect ratios. The former is in the order of 0.16 with very little variation between acidic and basic conditions and time. The latter, the shell aspect ratio, varies between $4.17(\mathrm{t}=0)$ and $3.80(\mathrm{t}=60 \mathrm{~min})$ at basic $\mathrm{pH}$ and 2.65 at acidic $\mathrm{pH}$. While we have previously reported ${ }^{18}$ that the core and shell aspect ratios can vary in OASL, the values found here seem to indicate that micelles can be described with an highly distorted oblate core (e.g., equatorial and axial core radii are, respectively, 2.56 $\mathrm{nm}$ and $0.46 \mathrm{~nm}$ for the basic system at $\mathrm{t}=0$ ) and highly pronounced oblate shell (e.g., equatorial and axial shell thicknesses are, respectively, $0.71 \mathrm{~nm}$ and $2.96 \mathrm{~nm}$ ). We then tend to assume that Model 1 seem to better describe the EASL micellar system. This hypothesis seems to be corroborated by the fact that the fit of the entire set of time-resolved data is possible using Model 1 with $\chi^{2} /$ Npts values between 0 and 5 (Figure S4d), while the same operation provides a highly heterogeneous set of data with poor $\chi^{2} / \mathrm{Npts}$ when Model 2 is used. To this regard, the modeldependent micellar parameters $\left(T_{S}, R_{c}, \rho_{S}\right.$ in Figure $\left.S 4 b, c\right)$ are practically constant with time in the basic system, in perfect agreement with the model-independent Rg evolution. Finally, Rg and $\left(\mathrm{R}_{\mathrm{c}}+\mathrm{T}_{\mathrm{S}}\right)$ are very close. 
Table 1 - Model-dependent structural parameters used to fit the time-resolved in-situ SAXS data presented in Figure 4C. A core-shell ellipsoid of revolution form factor model is used to fit the data. In Model 1, the core and shell aspect ratios are kept to 1, thus transforming the model in a core-shell sphere. In Model 2, the core and shell aspect ratios are set as free parameters. $\mathrm{R}_{\mathrm{c}}$ is the core radius, $\mathrm{T}_{\mathrm{S}}$ is the shell thickness, $\mathrm{s}$ is the shell SLD, aspect ratio (core) is the ratio between the longitudinal and equatorial $\left(\mathrm{R}_{\mathrm{c}}\right)$ core radii while aspect ratio (shell) is the ratio between the longitudinal and equatorial $\left(\mathrm{T}_{\mathrm{S}}\right)$ shell thickness.

$\begin{array}{lcccc} & \text { Model 1 } & & \\ \mathrm{pH} & & \text { Basic } & & \text { Acid } \\ \text { Time, min } & 0 & 30 & 60 & 0 \\ \mathrm{R}_{\mathrm{c}}, \mathrm{nm} & 1.16 & 1.16 & 1.15 & 1.13 \\ \mathrm{~T}_{\mathrm{S}}, \mathrm{nm} & 2.33 & 2.34 & 2.36 & 2.38 \\ \rho_{\mathrm{S}}\left(\mathrm{x} 10^{-4}\right), \mathrm{nm}^{-2} & 9.68 & 9.67 & 9.66 & 9.66 \\ \text { Aspect ratio (core) } & 1 & 1 & 1 & 1 \\ \text { Aspect ratio (shell) } & 1 & 1 & 1 & 1\end{array}$

\section{Model 2}

$\begin{array}{lllll}\mathrm{R}_{\mathrm{c}}, \mathrm{nm} & 2.56 & 2.71 & 3.03 & 2.42 \\ \mathrm{~T}_{\mathrm{S}}, \mathrm{nm} & 0.71 & 0.68 & 0.65 & 1.06 \\ \rho_{\mathrm{S}}\left(\mathrm{x} 10^{-4}\right), \mathrm{nm}^{-2} & 9.76 & 9.76 & 9.76 & 9.71 \\ \text { Aspect ratio (core) } & 0.18 & 0.16 & 0.13 & 0.17 \\ \text { Aspect ratio (shell) } & 4.17 & 4.25 & 3.80 & 2.65\end{array}$

The time-resolved in-situ SAXS analysis can be summarized as follows:

1) EASL form micelles having comparable shape and size both at acidic and basic $\mathrm{pH}(\mathrm{Rg}$ comprised between $3.3 \mathrm{~nm}$ and $3.5 \mathrm{~nm}$ ), in contradiction to what was found for the SASL system. ${ }^{18}$ 2) The structure of EASL micelles does not evolve with time, making them extremely stable objects. 3) The micellar-to-ribbon phase transition occurs much faster at acidic than at basic $\mathrm{pH}$. In this work, carried on under continuous flow, the stability of a pure micellar phase varies between less than 1 minute at acidic $\mathrm{pH}$ to about 40 minutes at basic $\mathrm{pH}$. These values may change under static conditions. 4) EASL micelles are overall larger (between 1.5 and 3 times according to $\mathrm{pH}$ ) than the corresponding micelles of OASL, or SASL, micelles. ${ }^{18}$ 5) If one assumes that Model 1 better describes the EASL micellar system, one should remark the larger contribution of the hydrophilic shell, if compared to OASL and SASL systems. 6) Considering the high stability of the EASL micelles, we make the hypothesis that the formation of twisted 

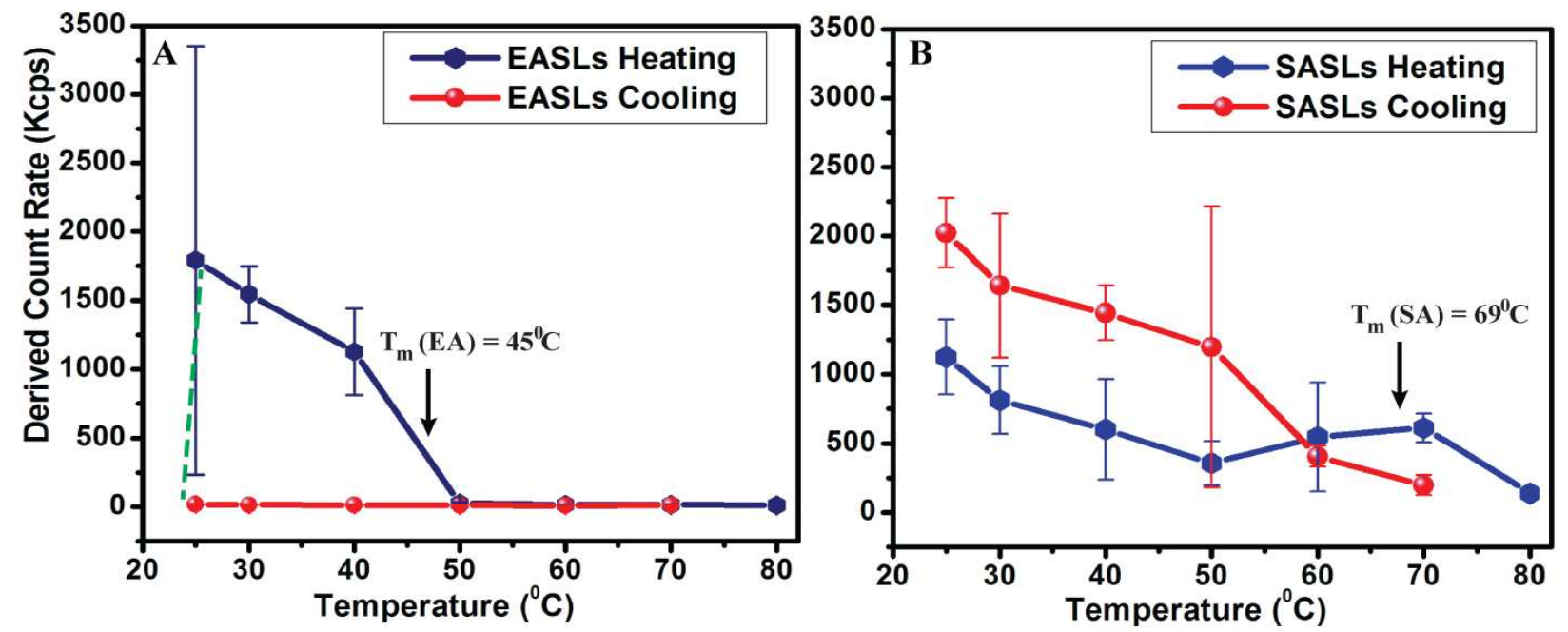

Figure 5: The results of the light scattering experiments at different temperatures on the self - assembled structures of (A) EASLs and (B) SASLs respectively. The dotted line represents the structure re formation of EASLs on standing.

To gain more insights into the structures formed by SASL and EASL and their stability, we looked at their temperature-dependent evolution. As the samples were heated in water, the EASLs twisted fibers started to disassemble around $\sim 45-50{ }^{\circ} \mathrm{C}$, after which the solution becomes clear, as indicated by the loss in scattered light (Figure 5A). Upon cooling to room temperature, the solution stays clear (Figure 5A), but a precipitate forms again after about 20 minutes at RT. Figure S5.A,B in the Supporting Information shows that the self - assembled structures before and after cooling are very similar as they both display a mixture of twisted, helical and flat fibers. The SASLs shows distinctly different behavior with respect to temperature. At room temperature and neutral $\mathrm{pH}$, the sample shows the formation of nanoscale twisted ribbons (Figure 2A), the light scattering signal of which is shown in Figure 5B. Upon heating, the self - assembled structures disassemble only in part, as shown by the residual light scattering signal at $\mathrm{T}>50^{\circ} \mathrm{C}$ and concomitant the turbidity is retained. After $\mathrm{T}=70{ }^{\circ} \mathrm{C}$, the solution becomes clear. While cooling, the scattered intensity starts to gradually increase again (Figure 5B) and, according to the corresponding morphological analysis using SEM (Figure $\mathrm{S} 5 \mathrm{D})$, one can observe similar twisted ribbons type of structures. Interestingly, the temperature 
stability of both EASL and SASL seems to depend on the corresponding temperature stability of their lipidic counterparts, as the melting temperature of elaidic and stearic acids are, respectively, $45^{\circ} \mathrm{C}$ and $69{ }^{\circ} \mathrm{C}$.

\section{Discussion:}

The summary of the experimental evidence described above is shown on Figure 6. Molecular self-assembly is driven by the minimization of free energy and several models have been developed in order to understand the relationship between the molecular architecture and the self-assembled structure..$^{20,53,54}$ The one developed by Israelachvili, Mitchell and Ninham puts in relationship the final aggregate morphology to the surfactant structure via the concept of a packing parameter, defined as $p=v_{0} /\left(a l_{0}\right)$, with $v_{0}$ and $l_{0}$ being respectively the volume and length of the surfactant tail and $a$ being the area per molecule, which, although influenced by the headgroup interactions, is in fact a thermodynamic quantity and it should be rather considered as an equilibrium area. The predictions based on the packing parameters $(p<1 / 3$ for spheres, $1 / 3$ $<p<1 / 2$ for cylinders, $p>1 / 2$ for bilayers) are fulfilled experimentally by a number of surfactant and lipid systems, but this model is not developed enough to explain the formation of twisted fibers, as this is the case in this work.

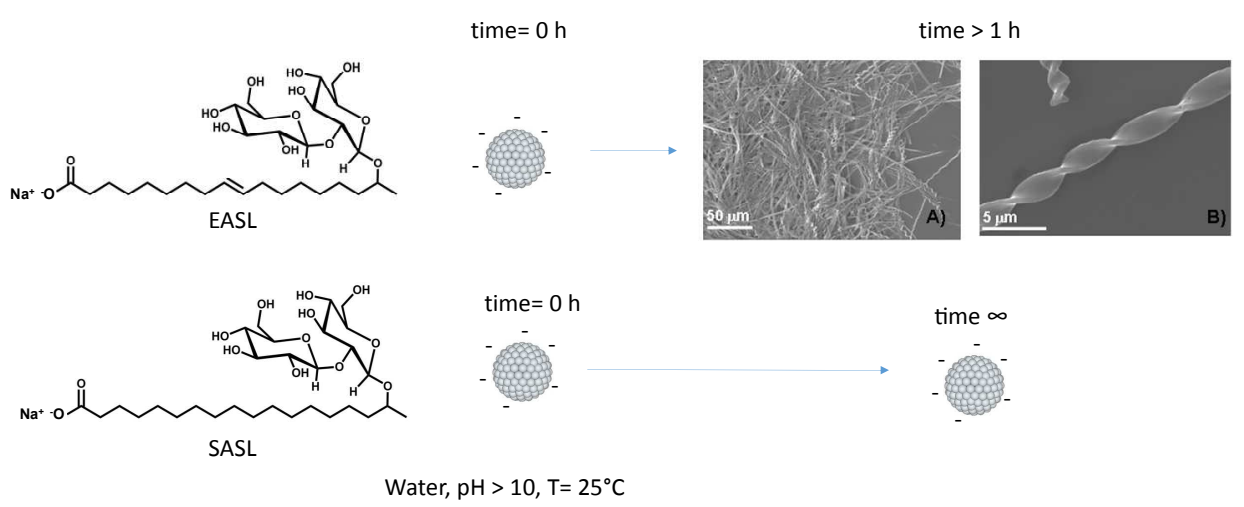

Figure 6: Summary of the self-assembly properties of EASL and SASL. At time $\mathrm{t}=0 \mathrm{~h}$ and basic $\mathrm{pH}$, both compounds form micelles. At longer time, EASL slowly forms giant twisted ribbons, while micelles are still the most stable form of SASL. The packing parameter for both molecules is estimated to be the same and about $0.28,{ }^{19}$ a value which supports the formation of micelles only.

Chiral fibers and tubes constituted of lipids are self-assembled structures which are nicely described by theories based on the minimization of the elastic free energy of a membrane, 

comprehensive review of T. G. Barclay et al. $)^{51}$ to better explain the large amount of apparently contradictory experimental data. In particular, twisted self-assembled fibers, similar to the ones observed in this work, have generally been considered as transients in the formation process of helical fibers and nanotubes; however, it is now well accepted that twisted fibers can also exist as stable equilibrium structures, a fact that is corroborated by experimental evidence in many systems and also explained from a theoretical point of view. ${ }^{51}$ According to these, ribbon edges, if not stabilized by their interactions with the solvent, tend to grow and eventually close into tubules. If edges are stabilized, for instance by hydration and electrostatic repulsion, ${ }^{56}$ ribbons, and even helices can stand as equilibrium structures. If the combination of the packing parameter model with the elastic theory approach could be of much help to understand, and even predict, the self-assembly process of twisted fibers in relationship with the lipid molecular structure, such a general approach has not been proposed, yet, and we must rely on both theories independently to propose an explanation for the different behavior of the EASL and SASL.

From a molecular point of view, the nature of the headgroup has been recognized to have a major impact on the ribbon formation with respect to the hydrophobic tail ${ }^{51}$ and we have ourselves shown that disaccharide (sophorose and cellobiose) headgroups combined with a saturated C18 fatty acid tail promote fiber formation, while mono-glucose headgroup promotes the formation of flat bilayers. ${ }^{18,19}$ These data are in line with the latest theories concerning chiral fiber formation and according to which fiber twisting can occur on the basis of a chiral symmetry breaking phenomenon of a membrane bilayer independently of molecular chirality, ${ }^{57,58}$ thus confirming the tight relationship between molecular geometry, packing and aggregate morphology for these complex structures. However, several authors, mainly in the study of the self-assembly properties of diacetylenic phospholipids, have discussed the impact of the hydrophobic tail configuration on the ribbon formation, possibly ascribed to the presence of a rigid, bent, tilt-inducing segment in the hydrophobic chain. ${ }^{51,59}$

The importance of the hydrophobic chain the self-assembly of surfactants was also highlighted by Nagarajan, ${ }^{60}$ who proposes an analytical expression to calculate the packing parameter $p$ taking into direct consideration the contribution of the surfactant tail, otherwise neglected. From a free energy point of view, he considers an additional packing contribution, which accounts for the packing constraints of the tail inside the aggregates but do not matter in 
the ideal case of a bulk hydrocarbon liquid phase. He then estimates the packing contribution using the so-called Uniform Concentration/Nonuniform Deformation Model, which takes into account the fact that the tail has to deform non uniformly along its length to fill the aggregate core with uniform density. Since the constraints of a sphere (high constraint), cylinder (high constraint in the end-caps and low constraints in the center) and bilayers (low constraints) are not the same, the contribution slightly differs for each morphology. The bottom line is that, if one expects, for a given surfactant molecule, the formation of a bilayer on the basis of the classical expression for $p$, in fact both cylinders and bilayers could occur, or even cylinders only, for a chain length going from 8 to 12 carbons (the polar headgroup being the same).

To understand the different behaviour of the self-assembly properties between EASL and SASL, which only differ in the presence of a double bond in the tail, all the rest being constant, one can combine Nagarajan hypotheses with the theoretical and experimental background of ribbon formation mentioned above. We have previously argued ${ }^{19}$ that the calculation of the packing parameter for acidic sophorolipids supports the formation of spherical micelles. At $\mathrm{pH}$ $\sim 11$, EASL and SASL do form micelles $(p<1 / 3)$, in agreement with the prediction. In the SASL case, spherical micelles do not undergo any phase change, as expected, although a minor fraction of SASL forms nanoscale platelets $(p=1)$ coexisting with spheroidal micelles under basic $\mathrm{pH}$ conditions. ${ }^{18}$ However, in the case of EASL, micelles slowly transform into flat twisted ribbons and eventually into helices, a fact which can only be explained by a time-dependent evolution of the packing parameter. Despite some similarities, the difference in the self-assembly behavior between SASL and EASL under basic pH conditions is remarkable and we believe that it can only be explained by considering the specific role of the trans double bond.

From a qualitative point of view, one must acknowledge that the presence of trans double bond impacts the chain rigidity and its ability to deform into a constrained spherical core. We have reported the micellar structures for acidic sophorolipids both at acidic and basic $\mathrm{pH}^{10,18}$ and it was put in evidence the important flexibility of the tail within the micelle, with possibility for micellar crossing and bending. At the moment, we do not know which deformation model could best describe bolaform amphiphiles like sophorolipids; to the best of our knowledge, the $\mathrm{COOH}$ (or $\mathrm{COO}^{-}$) group at the end of the tail preferentially sits at the micelle/solvent interface, rather than being in the micellar core, in oleic acid based sophorolipids. ${ }^{10,18}$ As far as the molecular distribution of SASL in twisted ribbons is concerned, solid state NMR arguments seem to 
support head-to-tail interdigitation among SASL, ${ }^{13}$ in line with previous works on lipid nanotubes prepared from bolaform glycolipids, ${ }^{61-63}$ while the interplanar distance value of 2.71 $\mathrm{nm}$ supports lipid bending within the ribbon plane. ${ }^{13}$ EASL fibers have an interplanar distance within the ribbon plane of $3.05 \mathrm{~nm}$, a size which is comparable to the EASL molecular length. This aspect indicates that: 1) the tilt angle, as described by Masuda and coworkers, ${ }^{61}$ of EASL in the plane is very small and 2) interdigitation can be the only possible configuration. Interestingly, the interplanar distance in EASL ribbons is systematically the same, at any $\mathrm{pH}$ value, thus suggesting that the molecular conformation within the ribbon plane is not affected by the protonation state of the carboxylic acid. Under these circumstances, it may not seem unreasonable that the presence of a stiff trans double bond in the middle of the tail reduces the tail flexibility and affects the local EASL packing at a point which the headgroup area, intended as an equilibrium area as described by Nagarajan, ${ }^{60}$ becomes low enough and the packing parameter actually increases and flat morphologies become more stable than highly curved ones like micelles. Concomitant $\pi$-interaction between the trans bonds can contribute to reduce the chain flexibility. If literature lacks data concerning the impact of the trans double bond on the self-assembly of fatty acids, our considerations seem to be in line with the work of Rappolt et al., ${ }^{61}$ who argued that differences (up to $26 \%$ ) in the expected stretching ratio between oleic and elaidic moieties in monoelaidin and monoolein-fatty acids based membranes could be one of the reasons for the deviation from the expected sphericity of lipids cages in the corresponding $F d 3 m$ mesophases. A recent review paper ${ }^{44}$ specifically highlights the role of trans geometry of membrane lipids in the bilayer fluidity and interactions with biological membrane lipids. For instance, lipids containing a trans double bond were shown to have a better affinity for cholesterol; the membrane displayed a higher sensitivity to temperature in the $20{ }^{\circ} \mathrm{C}-30{ }^{\circ} \mathrm{C}$ range, as the trans-lipids are rather in an ordered crystalline phase, if compared to their cis analogues, which are more fluid within the membrane; finally, the average diameter of a liposome, which is an indirect probe of a bilayer rigidity, was smaller for trans-phospholipids than for cis-phospholipids.

During the formation of flat twisted ribbons, the bulky sophorose disaccharide could be responsible for a chiral symmetry breaking phenomenon, just as it occurs in the formation of twisted ribbons at acidic $\mathrm{pH}$. Finally, it is interesting to mention the supposed double role of the $\mathrm{COOH}$ groups. At basic $\mathrm{pH}$, all EASL molecules are negatively charged, and attractive 
interactions favouring packing in the ribbon morphology are most likely balanced by the electrostatic repulsion between the COO-groups, which favours the micellar phase instead. The resulting ribbon formation kinetics is then much slower than at acidic $\mathrm{pH}$, at which $\mathrm{COO}$ - groups are mostly protonated and electrostatic repulsion minimized. Meanwhile, according to the current hypotheses explaining the ribbon formation, ${ }^{51,56} \mathrm{COO}$ - groups are necessary to stabilize the ribbon edges and prevent tube formation and aggregation.

\section{Conclusion:}

In conclusion, the helical structure formation of two geometrically similar sophorolipids was addressed. The EASLs form helical structures via micellar intermediate at all $\mathrm{pH}$ whereas the SASLs formed such structures under low $\mathrm{pH}$ conditions only. The structure formations were found to be slow for EASL whereas it is too rapid for SASL. Although the complete understanding is still not available, we believe that the possibly the $\pi-\pi$ interactions, or differences in the stretching of the fatty acid tail, between EASL and SASL are playing an important role in the helical structure formation, especially at basic $\mathrm{pH}$. On the other hand, the better hydrophobic stacking of stearic acid endows the helical structures from stearic acid SLs less vulnerable to heat and dissolution.

\section{Acknowledgements:}

This work was supported by Raman - Charpak Fellowship Scheme, thus the authors thanks CEFIPRA, DST - India and Campus France for the funding. P. D. thanks UGC - New Delhi (Govt. of India) for Senior Research Fellowship (SRF). We are grateful to Mr. Robin Marc, UPMC, College de France, Paris, France for his help in Raman experiments. P. D. and B. L. V. P. are grateful to Dr. A. A. Prabhune and Dr. C. V. Ramana (CSIR - NCL, Pune) for the SLs production and purification respectively. SAXS experiments were performed on beamline ID02 at the European Synchrotron Radiation Facility (ESRF), Grenoble, France.

\section{Supporting Information Available:}

NMR and Raman Spectra, photographs depicting the formation of self-assembled structures, the fitting details of the SAXS profiles and the self-assembled structures of EASL and SASL before 
and after heating the samples are provided as supporting information. This information is available free of charge via the Internet at http://pubs.acs.org/.

\section{References:}

(1) Aliprandi, A.; Mauro, M.; De Cola, L. Controlling and Imaging Biomimetic SelfAssembly. Nat Chem 2016, 8 (1), 10-15.

(2) Zhao, Q.; Lian, Z.; Gao, X.; Yan, Y.; Huang, J. General Approach To Construct Photoresponsive Self-Assembly in a Light-Inert Amphiphilic System. Langmuir 2016, 32 (45), 11973-11979.

(3) Hamley, I. W.; Castelletto, V. Self-Assembly of Peptide Bioconjugates: Selected Recent Research Highlights. Bioconjug. Chem. 2017, 28 (3), 731-739.

(4) Van Bogaert, I. N. A.; Saerens, K.; De Muynck, C.; Develter, D.; Soetaert, W.; Vandamme, E. J. Microbial Production and Application of Sophorolipids. Appl. Microbiol. Biotechnol. 2007, 76 (1), 23-34.

(5) Zhang, L.; Somasundaran, P.; Singh, S. K.; Felse, A. P.; Gross, R. Synthesis and Interfacial Properties of Sophorolipid Derivatives. Colloids Surfaces A Physicochem. Eng. Asp. 2004, 240 (1-3), 75-82.

(6) Develter, D. W. G.; Lauryssen, L. M. L. Properties and Industrial Applications of Sophorolipids. Eur. J. Lipid Sci. Technol. 2010, 112 (6), 628-638.

(7) Zhou, S.; Xu, C.; Wang, J.; Gao, W.; Akhverdiyeva, R.; Shah, V.; Gross, R. Supramolecular Assemblies of a Naturally Derived Sophorolipid. Langmuir 2004, 20 (19), 7926-7932.

(8) Chen, M.; Dong, C.; Penfold, J.; Thomas, R. K.; Smyth, T. J. P.; Perfumo, A.; Marchant, R.; Banat, I. M.; Stevenson, P.; Parry, A.; et al. Adsorption of Sophorolipid Biosurfactants on Their Own and Mixed with Sodium Dodecyl Benzene Sulfonate, at the Air/water Interface. Langmuir 2011, 27 (14), 8854-8866.

(9) Baccile, N.; Babonneau, F.; Jestin, J.; Pehau-Arnaudet, G.; Van Bogaert, I. Unusual, pHInduced, Self-Assembly of Sophorolipid Biosurfactants. ACS Nano 2012, 6 (6), 47634776.

(10) Manet, S.; Cuvier, A. S.; Valotteau, C.; Fadda, G. C.; Perez, J.; Karakas, E.; Abel, S.; Baccile, N. Structure of Bolaamphiphile Sophorolipid Micelles Characterized with SAXS, SANS, and MD Simulations. J. Phys. Chem. B 2015, 119 (41), 13113-13133.

(11) Dhasaiyan, P.; Pandey, P. R.; Visaveliya, N.; Roy, S.; Prasad, B. L. V. Vesicle Structures from Bolaamphiphilic Biosurfactants: Experimental and Molecular Dynamics Simulation Studies on the Effect of Unsaturation on Sophorolipid Self-Assemblies. Chem. - A Eur. J. 2014, 20 (21), 6246-6250.

(12) Dhasaiyan, P.; Le Griel, P.; Roelants, S.; Redant, E.; Van Bogaert, I. N. A.; Prevost, S.; Prasad, B. L. V; Baccile, N. Micelles versus Ribbons: How Congeners Drive the SelfAssembly of Acidic Sophorolipid Biosurfactants. ChemPhysChem 2017, 18, 643-652.

(13) Cuvier, A.-S.; Berton, J.; Stevens, C. V; Fadda, G. C.; Babonneau, F.; Van Bogaert, I. N. a; Soetaert, W.; Pehau-Arnaudet, G.; Baccile, N. pH-Triggered Formation of Nanoribbons from Yeast-Derived Glycolipid Biosurfactants. Soft Matter 2014, 10 (22), 3950-3959.

(14) Cuvier, A. S.; Babonneau, F.; Berton, J.; Stevens, C. V.; Fadda, G. C.; Genois, I.; Le Griel, P.; Péhau-Arnaudet, G.; Baccile, N. Synthesis of Uniform, Monodisperse, 
Sophorolipid Twisted Ribbons. Chem. - An Asian J. 2015, 10 (11), 2419-2426.

(15) Cuvier, A. S.; Babonneau, F.; Berton, J.; Stevens, C. V.; Fadda, G. C.; Péhau-Arnaudet, G.; Le Griel, P.; Prévost, S.; Perez, J.; Baccile, N. Nanoscale Platelet Formation by Monounsaturated and Saturated Sophorolipids under Basic pH Conditions. Chem. - A Eur. J. 2015, 21 (52), 19265-19277.

(16) Dhasaiyan, P.; Banerjee, A.; Visaveliya, N.; Prasad, B. L. V. Influence of the Sophorolipid Molecular Geometry on Their Self-Assembled Structures. Chem. Asian J. 2013, 8 (2), 369-372.

(17) Dhasaiyan, P.; Prasad, B. L. V. Self-Assembly of Bolaamphiphilic Molecules. Chem. Rec. 2017, 17 (6), 597-610.

(18) Baccile, N.; Cuvier, A.-S.; Prévost, S.; Stevens, C. V; Delbeke, E.; Berton, J.; Soetaert, W.; Van Bogaert, I. N. A.; Roelants, S. Self-Assembly Mechanism of pH-Responsive Glycolipids: Micelles, Fibers, Vesicles, and Bilayers. Langmuir 2016, 32 (42), 1088110894.

(19) Baccile, N.; Selmane, M.; Le Griel, P.; Prévost, S.; Perez, J.; Stevens, C. V.; Delbeke, E.; Zibek, S.; Guenther, M.; Soetaert, W.; et al. PH-Driven Self-Assembly of Acidic Microbial Glycolipids. Langmuir 2016, 32 (25), 6343-6359.

(20) Israelachvili, J. N.; Mitchell, D. J.; Ninham, B. W. Theory of Self-Assembly of Hydrocarbon Amphiphiles into Micelles and Bilayers. J. Chem. Soc. Faraday Trans. 2 1976, 72, 1525.

(21) Armon, S.; Efrati, E.; Kupferman, R.; Sharon, E. Geometry and Mechanics in the Opening of Chiral Seed Pods. Science. 2011, 333 (6050), 1726-1730.

(22) Forterre, Y.; Dumais, J. Generating Helices in Nature. Science. 2011, 333 (6050), 17151716.

(23) Gao, W.; Feng, X.; Pei, A.; Kane, C. R.; Tam, R.; Hennessy, C.; Wang, J. Bioinspired Helical Microswimmers Based on Vascular Plants. Nano Lett. 2014, 14 (1), 305-310.

(24) Zhao, M.-Q.; Zhang, Q.; Tian, G.-L.; Wei, F. Emerging Double Helical Nanostructures. Nanoscale 2014, 6 (16), 9339-9354.

(25) Qiao, Y.; Lin, Y.; Wang, Y.; Yang, Z.; Liu, J.; Zhou, J.; Yan, Y.; Huang, J. Metal-Driven Hierarchical Self-Assembled One-Dimensional Nanohelices. Nano Lett. 2009, 9 (12), 4500-4504.

(26) Li, L.; Jiang, H.; Messmore, B. W.; Bull, S. R.; Stupp, S. I. A Torsional Strain Mechanism To Tune Pitch in Supramolecular Helices. Angew. Chemie Int. Ed. 2007, 46 (31), 58735876.

(27) Yang, Y.; Suzuki, M.; Owa, S.; Shirai, H.; Hanabusa, K. Preparation of Helical Nanostructures Using Chiral Cationic Surfactants. Chem. Commun. 2005, No. 35, 44624464.

(28) Soto, C. Protein Misfolding and Disease; Protein Refolding and Therapy. FEBS Lett. 2001, 498 (2), 204-207.

(29) Soreghan, B.; Kosmoski, J.; Glabe, C. Surfactant Properties of Alzheimer's A Beta Peptides and the Mechanism of Amyloid Aggregation. J. Biol. Chem. 1994, 269 (46), 28551-28554.

(30) Lomakin, A.; Teplow, D. B.; Kirschner, D. A.; Benedek, G. B. Kinetic Theory of Fibrillogenesis of Amyloid B-Protein. Proc. Natl. Acad. Sci. 1997, 94 (15), 7942-7947.

(31) Mozaffarian, D.; Katan, M. B.; Ascherio, A.; Stampfer, M. J.; Willett, W. C. Trans Fatty Acids and Cardiovascular Disease. N. Engl. J. Med. 2006, 354 (15), 1601-1613. 
(32) Gebauer, S. K.; Psota, T. L.; Kris-Etherton, P. M. The Diversity of Health Effects of Individual Trans Fatty Acid Isomers. Lipids 2007, 42 (9), 787-799.

(33) Theodoratou, E.; McNeill, G.; Cetnarskyj, R.; Farrington, S. M.; Tenesa, A.; Barnetson, R.; Porteous, M.; Dunlop, M.; Campbell, H. Dietary Fatty Acids and Colorectal Cancer: A Case-Control Study. Am. J. Epidemiol. 2007, 166 (2), 181-195.

(34) Funari, S. S.; Barceló, F.; Escribá, P. V. Effects of Oleic Acid and Its Congeners, Elaidic and Stearic Acids, on the Structural Properties of Phosphatidylethanolamine Membranes. J. Lipid Res. 2003, 44 (3), 567-575.

(35) Yaghmur, A.; Sartori, B.; Rappolt, M. Self-Assembled Nanostructures of Fully Hydrated Monoelaidin - Elaidic Acid and Monoelaidin - Oleic Acid Systems. Langmuir 2012, 28 (26), 10105-10119.

(36) Roach, C.; Feller, S. E.; Ward, J. A.; Shaikh, S. R.; Zerouga, M.; Stillwell, W. Comparison of Cis and Trans Fatty Acid Containing Phosphatidylcholines on Membrane Properties. Biochemistry 2004, 43 (20), 6344-6351.

(37) Fameau, A.-L.; Arnould, A.; Saint-Jalmes, A. Responsive Self-Assemblies Based on Fatty Acids. Curr. Opin. Colloid Interface Sci. 2014, 19 (5), 471-479.

(38) Dowling, M. B.; Lee, J.-H.; Raghavan, S. R. pH-Responsive Jello: Gelatin Gels Containing Fatty Acid Vesicles. Langmuir 2009, 25 (15), 8519-8525.

(39) Valotteau, C.; Calers, C.; Casale, S.; Berton, J.; Stevens, C. V.; Babonneau, F.; Pradier, C. M.; Humblot, V.; Baccile, N. Biocidal Properties of a Glycosylated Surface:

Sophorolipids on Au(111). ACS Appl. Mater. Interfaces 2015, 7 (32), 18086-18095.

(40) Baccile, N.; Nassif, N.; Malfatti, L.; Van Bogaert, I. N. a.; Soetaert, W.; Pehau-Arnaudet, G.; Babonneau, F. Sophorolipids: A Yeast-Derived Glycolipid as Greener Structure Directing Agents for Self-Assembled Nanomaterials. Green Chem. 2010, 12 (9), 1564 1567.

(41) Kasture, M.; Singh, S.; Patel, P.; Joy, P. A.; Prabhune, A. A.; Ramana, C. V.; Prasad, B. L. V. Multiutility Sophorolipids as Nanoparticle Capping Agents: Synthesis of Stable and Water Dispersible Co Nanoparticles. Langmuir 2007, 23 (23), 11409-11412.

(42) Asmer, H. J.; Lang, S.; Wagner, F.; Wray, V. Microbial Production, Structure Elucidation and Bioconversion of Sophorose Lipids. J. Am. Oil Chem. Soc. 1988, 65 (9), 1460-1466.

(43) NMR data Elaidic Acid http://lipidlibrary.aocs.org/Analysis/content.cfm?ItemNumber=40261.

(44) Chatgilialoglu, C.; Ferreri, C.; Melchiorre, M.; Sansone, A.; Torreggiani, A. Lipid Geometrical Isomerism : From Chemistry to Biology and Diagnostics. Chem. Rev. 2014, 114 (1), 255-284.

(45) Cui, H.; Muraoka, T.; Cheetham, A. G.; Stupp, S. I. Self-Assembly of Giant Peptide Nanobelts. Nano Lett. 2009, 9 (3), 945-951.

(46) Hamley, I. W. Form Factor of Helical Ribbons. Macromolecules 2008, 41 (22), 89488950.

(47) Goldberger, J. E.; Berns, E. J.; Bitton, R.; Newcomb, C. J.; Stupp, S. I. Electrostatic Control of Bioactivity. Angew. Chemie - Int. Ed. 2011, 50 (28), 6292-6295.

(48) Treanor, R.; Pace, M. D. Microstructure, Order and Fluidity of 1,2-Bis(tricosa-10,12Diynoyl) -Sn-Glycero-3-Phosphocholine (DC8,9PC), a Polymerizable Lipid, by ESR and NMR. Biochim. Biophys. Acta 1990, 1046 (1), 1-11.

(49) Seung, H. S.; Nelson, D. R. Defects in Flexible Membranes with Crystalline Order. Phys. Rev. $A$ 1988, 38 (2), 1005-1018. 
(50) Brandow, S. L.; Turner, D. C.; Ratna, B. R.; Gaber, B. P. Modification of Supported Lipid Membranes by Atomic Force Microscopy. Biophys. J. 1993, 64 (3), 898-902.

(51) Barclay, T. G.; Constantopoulos, K.; Matisons, J. Nanotubes Self-Assembled from Amphiphilic Molecules via Helical Intermediates. Chem. Rev. 2014, 114 (20), 1021710291.

(52) Nagarajan, R. Self-Assembly of Bola Amphiphiles. Chem. Eng. Commun. 1987, 55, 251273.

(53) Tanford, C. The Hydrophobic Effect: Formation of Micelles and Biological Membranes; Wiley-Interscience, 1973.

(54) Nagarajan, R.; Ruckenstein, E. Theory of Surfactant Self-Assembly: A Predictive Molecular Thermodynamic Approach. Langmuir 1991, 7 (12), 2934-2969.

(55) Helfrich, W.; Prost, J. Intrinsic Bending Force in Anisotropic Membranes Made of Chiral Molecules Wolfgang. Phys. Rev. A 1988, 38 (6), 3065-3068.

(56) Boettcher, C.; Schade, B.; Fuhrhop, J. Comparative Cryo-Electron Microscopy of Noncovalent N -Dodecanoyl- ( D - and L - ) Serine Assemblies in Vitreous Toluene and Water. Langmuir 2001, 17, 873-877.

(57) Seifert, U.; Shillcock, J.; Nelson, P. Role of Bilayer Tilt Difference in Equilibrium Membrane Shapes. Phys. Rev. Lett. 1996, 77 (26), 5237-5240.

(58) Spector, M. S.; Easwaran, K. R.; Jyothi, G.; Selinger, J. V; Singh, A.; Schnur, J. M. Chiral Molecular Self-Assembly of Phospholipid Tubules: A Circular Dichroism Study. Proc. Natl. Acad. Sci. U. S. A. 1996, 93 (23), 12943-12946.

(59) Easwaran, K. R. .; Balasubramanlan, S. V.; Singh, A. Conformation of Polymerizable Diacetylenic Lecithin, DC8,9PC, by 1H-NMR Spectroscopy. Chem. Phys. Lipids 1994, 69, 161-165.

(60) Nagarajan, R. Molecular Packing Parameter and Surfactant Self-Assembly: The Neglected Role of the Surfactant Tail Self-Assembly. Langmuir 2002, 18, 31-38.

(61) Kameta, N.; Masuda, M.; Minamikawa, H.; Shimizu, T. Self-Assembly and Thermal Phase Transition Behavior of Unsymmetrical Bolaamphiphiles Having Glucose- And Amino-Hydrophilic Headgroups. Langmuir 2007, 23 (8), 4634-4641.

(62) Masuda, M.; Yoza, K.; Shimizu, T. Polymorphism of Monolayer Lipid Membrane Structures Made from Unsymmetrical Bolaamphiphiles. Carbohydr. Res. 2005, 340 (16), 2502-2509.

(63) Kameta, N.; Ishikawa, K.; Masuda, M.; Shimizu, T. Control of Self-Assembled Morphology and Molecular Packing of Asymmetric Glycolipids by Association/dissociation with Poly(thiopheneboronic Acid). Langmuir 2013, 29 (43), 13291-13298.

(64) Rappolt, M.; Cacho-Nerin, F.; Morello, C.; Yaghmur, A. How the Chain Configuration Governs the Packing of Inverted Micelles in the Cubic Fd3m-Phase. Soft Matter 2013, 9 (27), 6291-6300. 
Conditions that favor the helical structure formation in structurally similar sophorolipids (SLs), derived from elaidic acid (having a trans double bond between the C9 - C10 positions of the alkyl chain) and stearic acid (no double bond) are presented.

$313 \times 127 \mathrm{~mm}(120 \times 120 \mathrm{DPI})$ 